\title{
INOVAÇÃO DA GESTÃO ESTRATÉGICA COM A IMPLANTAÇÃO DO BALANCED SCORECARD
}

\section{STRATEGIC MANAGEMENT INNOVATION WITH THE IMPLEMENTATION OF THE BALANCED SCORECARD}

\author{
Mário de Carvalho Filho \\ Universidade Federal Rural de Pernambuco \\ Brasil. \\ ORCID: 0000-0002-8461-4645 \\ E-mail: mcf2508@hotmail.com \\ Tânia Nobre Gonçalves Ferreira Amorim \\ Universidade Federal Rural de Pernambuco \\ Brasil \\ ORCID: 0000-0002-9927-4241 \\ E-mail: tanobre@gmail.com
}

Recebido em: $25 / 04 / 2020$

Aprovado em:05/06/2020

\section{RESUMO}

A implantação do Balanced Scorecard (BSC) no modelo de gestão estratégica adotado pelo Ministério Público de Pernambuco (MPPE) foi objeto deste artigo, no período 2013-2016. Foi um estudo de caso em que foram utilizadas técnicas de pesquisa documental, análise de conteúdo e entrevistas com a equipe gestora. $\mathrm{O}$ referencial teórico escolhido abrange o balanced scorecard, características gerais e fatores de insucesso da implantação; exemplos do BSC no setor público e gestão estratégica. O MPPE foi exitoso no formato definido do BSC no seu modelo de gestão estratégica, pois a sequência de implantação seguida, adaptou-se à realidade da instituição. Na Gestão Estratégica 2013-2016, a metodologia BSC aplicada funcionou como ferramenta de gestão estratégia e como sistema de avaliação de desempenho. Também foi exitoso no seu modelo de gestão estratégica associado ao uso do BSC, porque nenhuma das causas de insucesso apontadas pela doutrina, na implantação do BSC foi identificada, talvez porque a sequência de implantação seguida foi adequada à realidade da instituição, apesar de alguns objetivos não terem sido realizados. O MPPE, pela primeira vez, teve um ciclo estratégico analisado desde sua construção até a execução, com todas as etapas registradas e analisadas e resultados alcançados. É uma iniciativa que foi premiada nacionalmente, com o Prêmio Innovare, que tem como objetivo identificar, divulgar e difundir práticas que contribuam para o aprimoramento da Justiça no Brasil. Mesmo assim, deverá ser aprimorada para o novo ciclo de gestão estratégica organizacional, como evolução natural e estratégica de gestão.

Palavras-chave: Balanced Scorecard. Gestão Estratégica. Setor Público. 


\begin{abstract}
The implementation of the Balanced Scorecard (BSC) in the strategic management model adopted by the Public Ministry of Pernambuco (MPPE) was the object of this article, in the period 2013-2016. It was a case study in which documentary research techniques, content analysis and interviews with the management team were used. The theoretical framework chosen covers the balanced scorecard, general characteristics and failure factors of the implementation; examples of the BSC in the public sector and strategic management. The $M P P E$ was successful in the BSC's defined format in its strategic management model, since the sequence of implementation followed, adapted to the reality of the institution. In the Strategic Management 2013-2016, the applied BSC methodology functioned as a strategy management tool and as a performance evaluation system. It was also successful in its strategic management model associated with the use of the BSC, because none of the causes of failure pointed out by the doctrine in the BSC implementation was identified, perhaps because the implementation sequence followed was adequate to the reality of the institution, although some objectives have not been carried out. The MPPE, for the first time, had a strategic cycle analyzed from its construction to execution, with all steps recorded and analyzed and results achieved. It is an initiative that has been awarded nationally, with the Innovation Prize, which aims to identify, disseminate and disseminate practices that contribute to the improvement of justice in Brazil. Even so, it should be improved for the new organizational strategic management cycle, as a natural evolution and strategic management.
\end{abstract}

Keywords: Balanced Scorecar. Strategic Management. Public Sector.

\title{
1 INTRODUÇÃO
}

Nas últimas décadas, as organizações públicas necessitaram mudar sua aplicação de recursos, por exigências como: transparência na prestação de contas, qualidade nos serviços e recursos orçamentários insuficientes. Cobra-se do gestor eficiência, eficácia e efetividade, seja ele de organização pública ou privada. Portanto, buscar aperfeiçoamento e profissionalização tem assumido papel fundamental. Para atender às novas demandas sociais, muitas organizações públicas passaram a adotar ferramentas e práticas gerenciais do setor privado, auxiliando no atingimento de metas e medição dos resultados (Peci, Pieranti \& Rodrigues, 2008). O tema desempenho organizacional vem recebendo cada vez mais atenção nas últimas décadas. No entanto, esta atenção é mais voltada para o setor privado, deixando lacunas de pesquisa na área pública (Gomes, Leal \& Assis, 2013).

Assim, o planejamento estratégico organizacional abrange definições de: missão, visão e valores; objetivos e metas da empresa; e o planejamento das estratégias (Odebrecht, Giarola, Silva, Martins \& LIMA, 2013). Souza e Marinho (2014), lembram que na implantação da estratégia há necessidade de reflexão sobre a situação da organização e quais serão os seus planos futuros, para definir seus planos de ação, sejam públicas ou privadas.

Uma das principais ferramentas gerenciais sugeridas para aprimorar a gestão estratégica e orçamentária da Administração Pública é o Balanced Scorecard - BSC, sabendo-se que são necessárias várias adaptações. A forma de construção do BSC varia porque Kaplan e Norton (1997; 2010), seus idealizadores, sugerem um plano de elaboração que pode ser adotado e adaptado por várias organizações, dividido em quatro processos gerenciais e dez etapas. É um sistema de alinhamento e controle estratégico baseado em 
indicadores de desempenho integrados (Niven, 2003; Félix, Félix \& Timóteo, 2011; Kartalis, Velentzas \& Broni 2013; Rosa, Petri, Petri \& Casagrande, 2014).

O Ministério Público de Pernambuco (MPPE) construiu o Manual de Gestão Estratégica 2013-2016, contendo o BSC como ferramenta de apoio gerencial. A pesquisa que originou este artigo respondeu a seguinte pergunta: como o modelo de gestão estratégica adotado pelo MPPE, no período de 2013-2016, relacionou-se com os objetivos estratégicos estabelecidos pela instituição? O objetivo deste artigo consistiu em conhecer a implantação do BSC no modelo de gestão estratégica adotado.

\section{BALANCED SCORECARD (BSC)}

O BSC, ou indicadores balanceados de desempenho, surgiu como uma proposta de mensuração estratégica estruturada complementar à avaliação tradicional de resultados, integrando indicadores financeiros e não financeiros, representando processos internos, relações empresa-clientes e capacidade de aprendizado e crescimento (Cunha \& Kratz, 2016).

A implantação do BSC é um fator sensível para alcançar sucesso. Silva, Santos e Prochnik (2008) estimam que o percentual de fracassos na implantação chega a $70 \%$. Entretanto, faz-se necessário, considerar também sua elaboração, porque cada organização possui estratégia específica e não existe forma padronizada para sua construção.

A construção do BSC normalmente inicia com a estratégia formulada, em conjunto com a visão e missão organizacional. Na sequência, são definidas perspectivas e identificados os objetivos estratégicos, seguidos pela escolha dos indicadores para cada objetivo, definição das metas de superação, ações para alcançá-las e a implantação, considerada um processo complexo e que não finda, pois o BSC é um processo contínuo (Wernke \& Bornia, 2001).

Kaplan e Norton (2010), idealizadores do BSC, definem que o processo de tradução da visão organizacional estabelece um entendimento único na organização, traduzindo a estratégia em termos operacionais, nos mapas estratégicos. O processo de comunicação conecta os objetivos departamentais aos individuais, comunicando a estratégia em toda a estrutura organizacional, além de identificar os processos-chave, estabelecendo medidas de desempenho. O processo de planejamento do negócio baseia-se nas metas estratégicas, alocando recursos e fixando prioridades. E o processo de feedback e aprendizado busca fornecer um aprendizado estratégico, para uma gestão estratégica ao longo do tempo.

Contudo, há divergências sobre o processo de implantação do $\mathrm{BSC}$, em relação ao planejamento estratégico. Moreira et al. (2012, p. 73) defendem a definição antecipada da estratégia, porque "não se pode fazer com que uma equipe chegue a um resultado se ela não entende qual o caminho a percorrer". Goldszmidt (2003) esclarece que a formulação da estratégia em conjunto com a implementação do BSC, tende a deixar o processo mais lento. Já Schnorrenberger, Gasparetto e Lunkes (2015) defendem a elaboração do planejamento estratégico durante o desenvolvimento do BSC. O mapa estratégico é uma representação gráfica para visualizar a estratégia organizacional, com uma cadeia de relações de causa e efeito entre objetivos estratégicos, metas e ações interligadas às perspectivas, essencial ao BSC (Anagnostopoulos \& Elmasides, 2010; Cunha \& Kratz, 2016). Em relação às etapas de construção do BSC, Kaplan e Norton (1997) sugerem as seguintes, lembrando que há flexibilidade organizacional para sua construção: 
1 - Escolha da unidade organizacional onde será implantado o BSC.

2 - Entrevistas com executivos para identificar as relações entre a unidade de negócio e a instituição.

3 - Realização de entrevistas com os executivos para comunicar o conceito de BSC.

4 - Síntese das informações para reunião com a alta administração.

5 - Realizar o primeiro workshop executivo, para buscar consenso em relação à missão e estratégia.

6 - Realizar reuniões com subgrupos, para refinar descrição dos objetivos estratégicos e identificar

indicadores que melhor representam os objetivos.

7 - Realizar o segundo workshop executivo, com a alta administração, subordinados diretos e um maior efetivo de gerentes de nível médio.

8 - Iniciar a implantação. Definir metas, planos de ação e responsáveis para coordenar a implantação da estratégia.

9 - Realizar o terceiro workshop executivo. Definir visão, objetivos e indicadores para acompanhar o desempenho organizacional, além das ações que serão adotadas para alcançarem as metas.

10 - Conclusão do plano de implantação do BSC.

Quadro 1. Etapas de construção do BSC.

Fonte: adaptado de Kaplan \& Norton, 1997.

Para organizações públicas, Osório (2003) propõe um modelo genérico de incorporação do BSC ao planejamento estratégico com dois momentos: elaboração e implantação. A elaboração divide-se em duas etapas: na primeira, com duas fases (preparação e apresentação da proposta de estudo para validação pela alta direção); na segunda, são analisados documentos, planejamento estratégico e construída uma versão preliminar do BSC. Concluída a elaboração, inicia-se a implantação, em duas etapas: na primeira, reuniões com a diretoria executiva para refinamento e validação do BSC. Na segunda, disseminação do BSC na organização e sua auto alimentação para continuidade ao processo.

A diferença entre o BSC da iniciativa privada e da pública consiste na adaptação das perspectivas, reconfiguração dos objetivos estratégicos e estabelecimento de metas. Deve definir e articular a execução de estratégias, favorecendo uma visão integrada dos objetivos, indicadores, metas e iniciativas (Dorweiler \& Yakhou, 2005; Fernandes, Furtado \& Ferreira, 2016).

O processo de implantação do BSC, contudo, apresenta altos índices de insucesso (Kaplan \& Norton, 2010; Wernke, Lunkes \& Bornia, 2002; Goldszmidt, 2003; Galas \& Forte, 2005; Filgueiras, Barros \& Gomes, 2010; Litvac, Calhau, Neto \& Oliva, 2010; e Moreira et al., 2012), devido a fatores organizacionais (processos e cultura organizacional, estratégia e sistema de informação) e ao processo de implantação e uso do BSC (liderança e apoio da alta administração, integração com processos gerenciais, equipe de projeto e dinâmica de aplicação do BSC). As principais causas de insucesso da implantação do BSC, a partir do levantamento bibliográfico, são: 


\begin{tabular}{|l|}
\hline Falta de comprometimento e apoio da alta administração \\
\hline Envolvimento de poucas pessoas \\
\hline Encastelamento no topo organizacional \\
\hline Processos de desenvolvimento muito longos \\
\hline Tratamento como projeto da área de sistemas \\
\hline $\begin{array}{l}\text { Contratação de consultores inexperientes ou não } \\
\text { contratação }\end{array}$ \\
\hline Implantação apenas para fins de remuneração \\
\hline Cultura organizacional resistente a mudanças \\
\hline Ausência de uma cultura de planejamento estratégico \\
\hline Complexidade do negócio e do projeto \\
\hline Estilo de gestão centralizador e autoritário \\
\hline $\begin{array}{l}\text { Restrição de tempo dos gestores para se dedicarem ao } \\
\text { projeto }\end{array}$ \\
\hline Falta de suporte de um sistema de informação \\
\hline Não realização de treinamento com os participantes \\
\hline
\end{tabular}

Quadro 2. Principais causas e fatores de insucesso da implantação do BSC Fonte: elaborado pelos autores

\section{MODELOS DE IMPLANTAÇÃO DO BSC NO SETOR PÚBLICO}

Cada organização desenvolve metodologia própria de implantação do BSC, dado o alto índice de insucesso (Silva, Santos \& Prochnik, 2008). Pessoa (2000), em sua tese de doutorado, desenvolveu um modelo de gestão baseado no BSC focado nas universidades públicas federais brasileiras, com objetivo estimular a racionalização dos recursos, com duas fases de implantação: preparação e operacionalização e 8 passos:

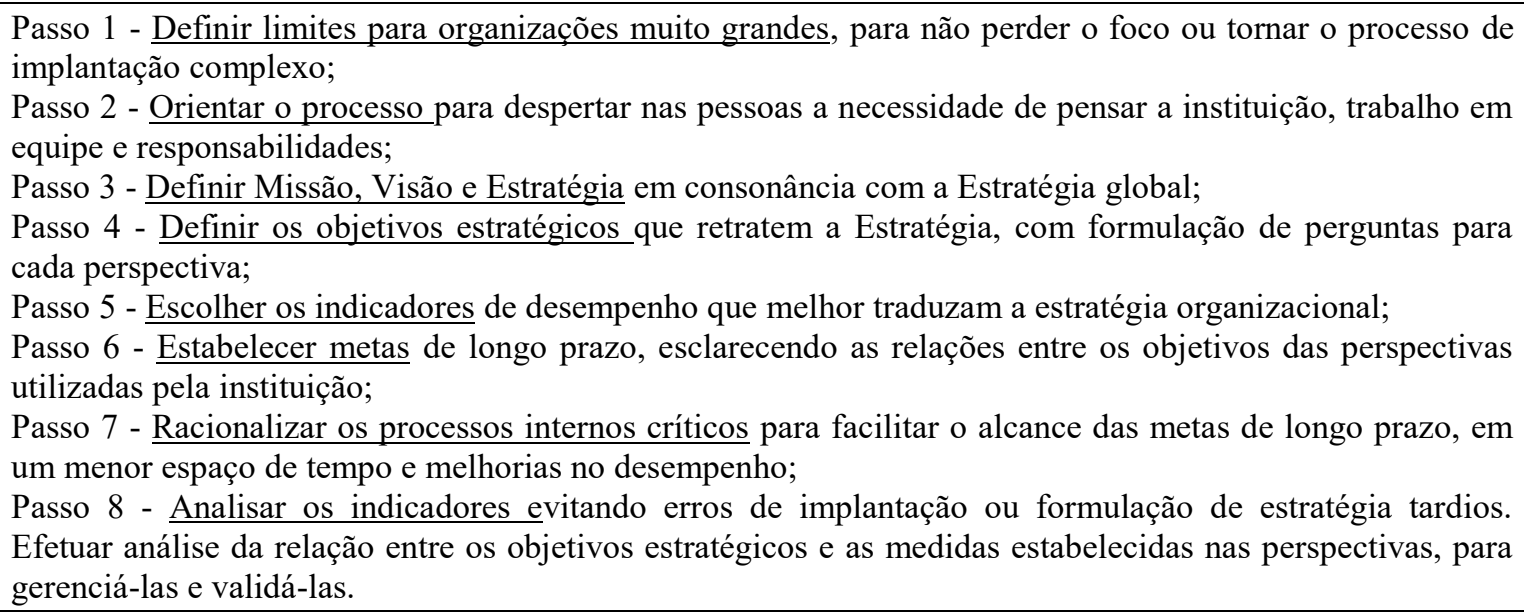

Quadro 3. Passos para implantação do BSC

Fonte: Silva, Santos \& Prochnik (2008)

Muscat, Biazzi \& Miguel (2007), ao descreverem o processo de formulação e implantação do BSC numa instituição pública de ensino, sugeriram 6 (seis) passos, semelhantes aos anteriores, com mais detalhes na definição, orientação e construção do mapa estratégico, com indicadores e iniciativas prioritárias. 
Passo 1 - Definir a orientação estratégica: estabelecida a visão da organização;

Passo 2 - Identificar os temas-chave que conduzem à estratégia, com uma consultoria externa, montadas equipes: de líderes; líderes agregados e de desenvolvimento, para elaborar objetivos estratégicos preliminares;

Passo 3 - Construir o mapa estratégico adaptado à realidade organizacional;

Passo 4 - Determinar indicadores e metas: com ênfase nos indicadores estratégicos, sem esquecer os operacionais;

Passo 5 - Selecionar iniciativas prioritárias: realizando workshops para captar sugestões;

Passo 6 - Estabelecer plano de implantação.

Quadro 4. Processo de formulação e implantação do BSC

Fonte: adaptado de Muscat, Biazzi \& Miguel (2007)

Muller (2001) também propôs um modelo de gerenciamento estratégico com o BSC para uma Instituição de Ensino Superior (IES), sem fins lucrativos, com sete passos semelhantes ao modelo anterior, mas que atende às peculiaridades da organização estudada. Já Osório (2003), propôs a implementação do BSC, buscando integrar o planejamento estratégico do setor público ao contexto da Nova Gestão Pública, com quatro etapas e oito fases.

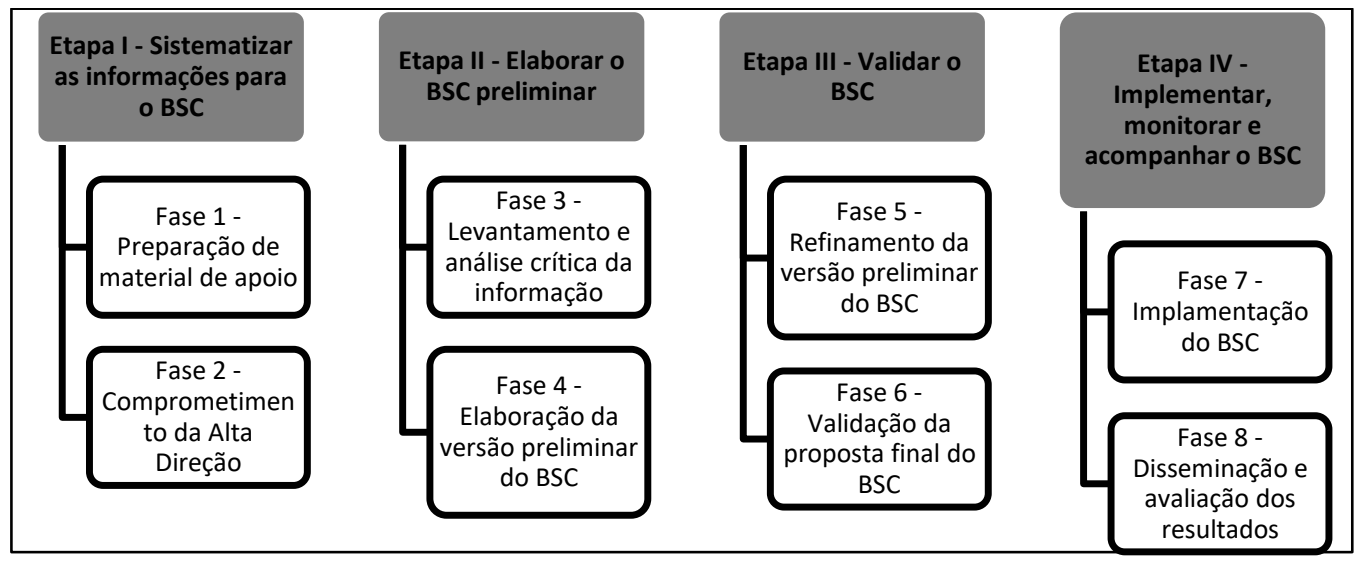

Figura 1. Modelo de implantação do BSC no Setor Público por Osório (2003) Fonte: adaptado de Osório (2003)

A descrição desses exemplos do processo de implantação do BSC em organizações públicas detalha diferentes possibilidades de adaptação desta ferramenta gerencial, criada para o setor privado. Confirma-se que diversas adaptações precisam ser realizadas, a partir da realidade organizacional, pessoal e gestão estratégica idealizada. Contudo, são identificadas semelhanças entre si. O BSC em organizações públicas melhora a administração destas, pois a definição das perspectivas e indicadores de desempenho auxiliam sobremaneira o entendimento e clarificação da gestão realizada, associada aos resultados alcançados.

Northcott \& Taulapapa (2012) elencaram os seguintes fatores de sucesso para a implementação do BSC no setor público: comprometimento da alta administração; ênfase na excelência do desempenho; realização de treinamentos, estratégia e metas organizacionais claras; e aplicação adequada de programas de incentivo e financiamento. Pode-se perceber que estes fatores não consistem em exclusividade do setor público, ocorrendo também na iniciativa.

Assim, o BSC é a ferramenta sugerida e a mais indicada para aprimorar a gestão estratégica e orçamentária dos entes da Administração Pública e proporciona instrumentos para mensuração do desempenho de seus programas (ROSA, et al., 2014). Corroborando com 
estes autores, Vargas, Diehl, Ayres e Monteiro (2015) também afirmam que o método mais difundido para medir os indicadores de desempenho não financeiros é o BSC, pois o mesmo permite o desenvolvimento de relações causais entre objetivos de longo prazo e curto prazo.

\section{METODOLOGIA}

A pesquisa realizada quanto aos seus objetivos foi descritiva. Em relação à estratégia, foi utilizado o estudo de caso, com abordagem qualitativa, e a coleta dos dados foi feita através de pesquisa documental e entrevistas. Martins \& Theóphilo (2017) esclarecem que a pesquisa documental é frequente em estudos de caso por fornecer grande material para estudo e análises aprofundadas. Em relação à pesquisa qualitativa, Vieira (2010) destaca que é predominantemente indutiva, ou seja, não se utiliza da prática de testar hipóteses, o que, pode interferir na capacidade de observação do pesquisador. Creswell (2014) esclarece que a pesquisa qualitativa utiliza diversas estratégias de investigação, métodos de coleta e análise de dados, que se baseiam em dados de textos e imagens extraídos da realidade organizacional, como foi realizado neste estudo.

Em relação às técnicas de análise de informações, as informações coletadas foram analisadas por meio da análise de conteúdo, com categorização dos elementos constitutivos dos documentos organizacionais e relatos das entrevistas. A escolha dos documentos para análise foi baseada nos objetivos do trabalho. Terminada esta fase, foram detalhadamente estudados, catalogados e absorvidos os elementos essenciais dos documentos. Posteriormente foram realizadas três entrevistas semiestruturadas com os gestores da área de Planejamento do MPPE, com o objetivo de compreender situações não esclarecidas pela pesquisa documental e, buscar o ponto de vista dos que participaram diretamente na gestão estratégica organizacional.

A pesquisa buscou responder a seguinte pergunta: como o modelo de gestão estratégica adotado pelo MPPE, no período de 2013-2016, se relacionou com os objetivos estratégicos estabelecidos pela instituição? O objetivo desta pesquisa consistiu em conhecer a implantação do BSC no modelo de gestão estratégica adotado.

Historicamente, desde a colonização do Brasil por Portugal, a nomenclatura de procurador-geral aparecia entre documentos da coroa. Ao longo do desenvolvimento político do Brasil, o Ministério Público foi avançando em termos de instituição, oscilando entre subordinação ao Poder Executivo e ao Poder Judiciário. Foi por meio da promulgação da Constituição Federal Brasileira de 1988 que se tornou independente. O Ministério Público Brasileiro é dividido em Ministérios Públicos Estaduais e Ministério Público da União. Este último dividido em Ministério Público Federal, Ministério Público Militar, Ministério Público do Trabalho e Ministério Público do Distrito Federal e Territórios.

Especificamente em Pernambuco, o Ministério Público de Pernambuco - MPPE, lócus da pesquisa, encontra-se organizado nos termos da Lei Orgânica Estadual (Lei Complementar $\mathrm{n}^{\circ} 12$, de 27/12/1994, com alterações posteriores). Trabalha pela cidadania e pela Justiça Social no Estado, atuando para que os direitos da população sejam respeitados pelas empresas, agentes públicos e próprios cidadãos, assim como para que sejam cumpridas as leis, pois a Instituição é fiscal desse cumprimento (Manual De Gestão Estratégica 2013/2016 MPPE, 2014, P. 14).

\section{ANÁLISE E DISCUSSÃO DOS RESULTADOS}

O Ministério Público de Pernambuco (MPPE), desde 1999, busca a melhoria na utilização de ferramentas de gestão que possam colaborar com as áreas técnicas e 
operacionais, tornando-as mais qualificadas no desenvolvimento de suas atividades. Desta forma, dois documentos deram início à construção da gestão estratégica para o ciclo 20132016: o projeto executivo e a revisão documental.

No projeto executivo, são encontrados elementos como público alvo, escopo do trabalho e o cronograma de atividades que foi desenvolvido para a elaboração da gestão estratégica do MPPE. Assim, faz parte do seu conteúdo: a elaboração da estratégia; método de trabalho e; plano de trabalho envolvendo um cronograma detalhado das atividades a serem executadas e seus respectivos períodos (Projeto Executivo MPPE, 2012).

Foi contratada uma consultoria para apoiar na gestão estratégica, sendo responsável pelo planejamento estratégico do MP nacional, e foram repassados para ela diversos documentos que demonstraram as peculiaridades internas e externas da organização, com destaque para sua cultura, perfil das lideranças e servidores e, modelo de gestão utilizado pela instituição (MPPE em Foco, 2012). Assim, o processo de implantação do BSC no MPPE cumpriu uma das exigências mais destacadas pelos autores estudados com fator de insucesso na implantação da ferramenta, que é a ausência de uma consultoria experiente participando do processo (Kaplan \& Norton, 2010; Litvac et al., 2010 e; Moreira et al., 2012). Em relação aos exemplos buscados no setor público, aparentemente, apenas Muscat, Biazzi \& Miguel (2007) observaram a importância deste cuidado.

Nesse processo, inicialmente, a consultoria analisou os seguintes documentos: Planejamento Estratégico 2005-2008; Planejamento Estratégico 2009-2012; Relatórios de Gestão; Planos Operacionais; Relatórios de Planejamento Estratégico; Fluxo de Monitoramento do Plano Estratégico; Regulamento das atribuições; Pesquisas; Projetos de Modernização; Projetos vinculados à atividade finalística do MPPE; Publicações diversas, Site e Portal de Internet do MPPE; e Pensamento e opiniões internas (MPPE em foco, 2012).

Inicialmente, a proposta consolidada entre a consultoria e o MPPE não previa a participação da sociedade na produção da gestão estratégica da instituição. Entretanto, entendeu-se que, por se tratar de uma organização pública, seria fundamental a "escuta social" trazendo a sociedade para participar da implantação do planejamento estratégico. Isto foi destacado por Muller (2001), quando descreve no seu estudo, a realização de dois seminários, nos quais, participaram representantes das comunidades interna e externa à instituição, interessados no seu desempenho institucional.

Com isso, foram acrescidas ao projeto executivo Fóruns de Gestão nas cidades de Cabo de Santo Agostinho, Recife, Olinda, Salgueiro, Garanhuns e Gravatá, em Pernambuco, Brasil. A ideia foi colocar frente a frente, membros e servidores da instituição, com a sociedade civil organizada, com o objetivo de entender as necessidades sociais destas localidades.

O primeiro Fórum consistiu em uma pesquisa de opinião pública sobre a percepção da sociedade em relação ao MPPE. O segundo foi uma pesquisa de clima organizacional dirigida aos procuradores de Justiça, promotores e demais servidores. E o terceiro foi realizado um diagnóstico organizacional, que coletou informações sobre temas relacionados com as funções exercidas pelos integrantes do MPPE (Revista MPPE em foco, 2012). As principais demandas foram a Defesa do direito à Saúde, o Combate à Criminalidade e a Defesa da Criança e do Adolescente, que compuseram os objetivos estratégicos do MPPE no ciclo 2013-2016. 


\begin{tabular}{|l|c|c|c|c|c|c|}
\hline \multirow{2}{*}{ Demandas Prioritárias } & \multicolumn{5}{|c|}{ Cidades do Estado de Pernambuco - Brasil } \\
\cline { 2 - 8 } & $\begin{array}{c}\text { Cabo de } \\
\text { Sto. } \\
\text { Agostinho }\end{array}$ & Olinda & Recife & Gravatá & Salgueiro & Garanhuns \\
\hline Defesa do direito à saúde & $20 \%$ & $18 \%$ & $13 \%$ & $18 \%$ & $13 \%$ & $12 \%$ \\
\hline Combate à criminalidade & - & $10 \%$ & $13 \%$ & $19 \%$ & $14 \%$ & $18 \%$ \\
\hline $\begin{array}{l}\text { Defesa da criança e do } \\
\text { adolescente }\end{array}$ & $15 \%$ & $14 \%$ & $10 \%$ & $14 \%$ & $12 \%$ & $17 \%$ \\
\hline Defesa da educação & $11 \%$ & $6 \%$ & $13 \%$ & - & $8 \%$ & $12 \%$ \\
\hline Defesa do meio ambiente & $8 \%$ & $3 \%$ & $6 \%$ & $7 \%$ & $10 \%$ & $4 \%$ \\
\hline Defesa da pessoa idosa & - & $10 \%$ & $6 \%$ & $8 \%$ & $6 \%$ & $8 \%$ \\
\hline Combate à corrupção & - & $10 \%$ & $8 \%$ & - & $7 \%$ & $8 \%$ \\
\hline $\begin{array}{l}\text { Defesa do direito à Segurança } \\
\text { pública }\end{array}$ & - & $7 \%$ & - & $9 \%$ & $7 \%$ & $8 \%$ \\
\hline $\begin{array}{l}\text { Defesa da pessoa com } \\
\text { deficiência }\end{array}$ & - & $4 \%$ & - & - & $4 \%$ & $3 \%$ \\
\hline Defesa do consumidor & - & $2 \%$ & - & - & $3 \%$ & $1 \%$ \\
\hline Defesa do Patrimônio Público & - & $2 \%$ & - & - & $2 \%$ & $1 \%$ \\
\hline Combate à sonegação fiscal & - & $2 \%$ & - & - & $3 \%$ & $1 \%$ \\
\hline $\begin{array}{l}\text { Defesa da Habitação e } \\
\text { urbanismo }\end{array}$ & - & $1 \%$ & - & - & $2 \%$ & $1 \%$ \\
\hline Defesa do Patrimônio Histórico & - & $1 \%$ & - & - & - & - \\
\hline Defesa dos direitos Humanos & - & - & $11 \%$ & - & $6 \%$ & $5 \%$ \\
\hline
\end{tabular}

Quadro 5. Resumo das demandas prioritárias identificadas nos Fóruns de Gestão Fonte: Adaptado da Revista MPPE em FOCO, 2012.

As etapas do BSC no MPPE, em síntese, foram as seguintes:

\begin{abstract}
Etapa 1 - Organização do trabalho: Nesta etapa, foram realizadas duas atividades. A elaboração do projeto executivo, documento que deu início à construção do planejamento estratégico do MPPE, para o período 2013-2016 e, a revisão documental, atividade que proporcionou uma visão geral da instituição para a consultoria contratada para implantação do BSC.

Etapa 2 - Visão de lideranças: mobilização das lideranças por meio de palestras a diferentes grupos, sobre gestão estratégica. Entrevistas com as lideranças, envolvendo público interno e externo, com o objetivo de captar a visão destes públicos sobre a instituição.

Etapa 3 - Mapa Estratégico: Para a construção do mapa estratégico realizou, com base no resultado das entrevistas, um seminário de dois dias, com a participação do grupo decisório da organização. Nesta etapa, também foram definidas a missão, visão e valores do MPPE.

Etapa 4 - Referências Estratégicas: Esta denominação utilizada pelo MPPE, faz referência à missão, visão e os valores da instituição. Nesta etapa foram elaboradas duas das principais ferramentas utilizadas na execução da gestão estratégica 2013-2016: o painel de gestão que reúne os indicadores de desempenho que medirão o resultado dos objetivos existentes no mapa estratégico. Os projetos estratégicos representam uma inovação na execução da gestão estratégica, pois cada projeto estratégico consiste em colocar em prática atividades que auxiliem o MPPE a alcançar sua missão.

Etapa 5 - Desdobramentos: Três atividades foram realizadas nesta etapa. Curso sobre BSC para nivelar o conhecimento sobre a ferramenta por toda organização. Estabelecimento de metas setoriais para incentivar todos os setores a seguirem numa direção única. Aumento da eficiência nas atividades internas e externas do MPPE pela adoção de processos estratégicos.

Etapa 6 - Gestão e comunicação: O modelo de Gestão projetado para o MPPE, no período 2013-2016, foi pensado para subsidiar a tomada de decisão da instituição. Neste sentido, o projeto executivo previu a realização de oficinas de planejamento com os líderes da organização. Implementado o Modelo de Gestão, as reuniões de avalição da estratégia foram criadas para realizarem o acompanhamento do planejamento estratégico, assim como, a sua evolução e maturidade.
\end{abstract}

Quadro 6. Etapas do BSC no MPPE

Fonte: Dados da pesquisa 
O projeto executivo previu dois eventos para públicos diferentes: uma palestra sobre gestão estratégica, com foco nas lideranças internas e externas da organização, que tratou da utilização do BSC na administração pública, e um fórum de gestão estratégica, no qual participaram diversos setores da sociedade que contribuíram para formulação e aprimoramento do diagnóstico estratégico do MPPE.

Após a realização das entrevistas com atores internos e externos ao MPPE, foi possível identificar a percepção do público interno e externo em relação aos desafios estratégicos enfrentados pelo MPPE para o período de 2013-2016. Com base nestas respostas, foram identificados pontos de convergência relacionados aos temas das seguintes dimensões: retorno para a sociedade; resultados institucionais; processos internos; pessoas; infraestrutura e tecnologia e; financeira e orçamentaria. Assim, foi elaborada uma proposta de mapa estratégico que traduziria os desafios para o MPPE no horizonte 2013-2016 (Projeto Executivo MPPE, 2012).

De acordo com o projeto executivo (2012, p. 13) “o Mapa Estratégico é na verdade o coração da estratégia organizacional. Nele os desafios são traduzidos em forma de objetivos estratégicos que representam grandes conquistas da instituição na direção do alcance da visão de futuro". Inicialmente, a equipe de desenvolvimento da gestão estratégica do MPPE, juntamente com a equipe da consultoria, elaborou um mapa estratégico preliminar, o qual deveria estar alinhado com as orientações das lideranças e possuir as seguintes perspectivas: resultados institucionais; processos internos; pessoas; infraestrutura e tecnologia; e orçamentária e financeira. Em seguida, as lideranças entrevistadas validaram o mapa estratégico em uma oficina de planejamento, realizada em 2012 ainda na fase de construção do planejamento estratégico.

Em meio a esse processo de construção do mapa estratégico do MPPE, foi definido qual seria o referencial estratégico da instituição para o período 2013-2016. Com isto, ficaram estabelecidos: missão, visão e valores do MPPE. Membros e servidores, da capital e do interior, em conjunto com a empresa de consultoria, auxiliaram e opinaram. Já os valores institucionais foram escolhidos por meio de uma votação na intranet ministerial, por meio de um questionário com doze sugestões, no qual, o integrante do MPPE poderia escolher quatro palavras chave (MPPE em Foco, 2012).

Nesse sentido, percebe-se que o MPPE optou por implantar o BSC paralelamente com a definição de sua estratégia utilizada para o ciclo 2013-2016. Não seguindo o que Wernke e Bornia (2001) e Moreira et al. (2012) defendem de que a construção do BSC deve ser sempre realizada com a estratégia já implantada, em conjunto com a visão e declaração de missão da organização.

Definido o mapa estratégico, a organização permitiu que as unidades e servidores estivessem alinhados e compromissados com o referencial estratégico da instituição. Esta conexão foi garantida por meio do mapa estratégico, que consiste no meio de comunicação interna que demonstra como as ações do MPPE se converterão em resultados e que contribuirão para o cumprimento da missão da organização (MPPE em Foco, 2012). Esta fase atende ao segundo processo gerencial sugerido por Kaplan e Norton (1997), comunicação e ligação, que possui a finalidade de conectar os objetivos departamentais aos individuais, comunicando a estratégia de uma ponta a outra da estrutura da instituição além de identificar os processos-chave, estabelecendo suas medidas de desempenho.

O Painel de Gestão e a lista de projetos estratégicos foram considerados peças fundamentais para a implantação da gestão estratégica do MPPE. O documento propõe a realização de uma reunião de repasse da metodologia para grupos de trabalho que tiveram a missão de: descrever os objetivos estratégicos; elaborar a ficha dos indicadores; e definir uma 
lista de projetos estratégicos. Todo este processo foi acompanhado pela consultoria, que pôde fazer sugestões, caso fosse necessário. Pode-se observar nesta fase de implantação da metodologia BSC, no MPPE, semelhanças com parte dos processos de implantação adotados por Pessoa (2000), Wernke e Bornia (2001) e, Gallon, Nuintin e Nogueira (2010), em relação à definição dos objetivos estratégicos, a escolha dos indicadores utilizados $\mathrm{e}$ ao estabelecimento das metas.

Fazem parte do Painel de Gestão os indicadores de desempenho e as metas. O projeto executivo explica que a quantidade de indicadores, por objetivo estratégico, deve obedecer a proporção de 1,5 indicador para cada objetivo estratégico. Cada indicador possui: título; descrição; fórmula de cálculo; polaridade; fonte de dados; responsável; e linha de base do indicador. Em relação às metas, o projeto executivo esclarece que todo indicador possui um nível desejado de desempenho que deve ser buscado pela instituição durante os anos de 2013, 2014, 2015 e 2016. Estas metas devem ser elaboradas levando-se em conta fatores contextuais e a linha de base, quando houver.

A escolha dos indicadores utilizados pelo MPPE, para o acompanhamento da gestão estratégica 2013-2016, teve a participação efetiva de membros e servidores da instituição, que se reuniram em dois dias, nos mesmos moldes que definiram a missão, visão e o mapa estratégico da organização. A Revista MPPE em FOCO (2012), Edição Extra que trata apenas de planejamento estratégico, afirma que o sucesso da Gestão Estratégica 2013-2016 do MPPE está nos indicadores, pois por meio deles será possível realizar a medição das metas definidas pela instituição e, com isso promover o controle, o acompanhamento e a revisão das ações. Declara também a revista que:

Os indicadores nascem para atender um objetivo e tomam como base o mapa estratégico da instituição. Com eles será possível embasar a análise crítica dos resultados obtidos, contribuir para a revisão do processo de Gestão Estratégica, permitindo a melhoria dos processos organizacionais, além de facilitar planejamento e controle do desempenho (Revista MPPE em Foco, 2012, p. 20)

O projeto executivo, elaborado pela empresa de consultoria para dar início à elaboração da gestão estratégica do MPPE para o quadriênio 2013-2016, apresentou duas ferramentas fundamentais na implantação da gestão estratégica da instituição. A primeira consistiu no Portfólio de Projetos Estratégicos que, segundo o projeto executivo MPPE (2012, p. 15) "Trata-se de um conjunto de projetos com alto grau de contribuição para a estratégia". Salienta-se que algumas atividades foram consideradas para a definição do portfólio, dentre elas: realizar a classificação dos projetos quanto à intensidade de gestão (baixa, moderada ou alta); verificar o alinhamento dos projetos aos objetivos estratégicos e; realizar a programação estratégica dos projetos. A segunda ferramenta foram as Reuniões de Avaliação da Estratégia (RAE), que foram utilizadas periodicamente, após a escolha do modelo estratégico adotado pela instituição, para o acompanhamento e avaliação da sua estratégia.

\section{CONSIDERAÇÕES FINAIS}

Ao se estudar o Planejamento Estratégico em organizações públicas, verifica-se que muitas iniciativas estão se aproximando da realidade da iniciativa privada, em especial o uso de ferramentas como o Balanced Scorecard, servindo de apoio em todas as etapas da gestão estratégica organizacional. O BSC é sugerido para aprimorar a gestão estratégica e orçamentária da Administração Pública e proporciona instrumentos para mensuração do desempenho de seus programas. 
Não existe uma forma padronizada ou correta para implantação do BSC, contudo, há fatores influenciadores do seu insucesso, em relação às perspectivas utilizadas, sequência para a implantação, bem como diversos aspectos organizacionais ligados à sua cultura, gestores, trabalhadores, processo de comunicação interna e externa e o próprio processo de elaboração do planejamento estratégico.

O BSC, como ferramenta de apoio à gestão estratégica e medição de desempenho, exige um estudo preliminar e profundo, sobre a organização que deseja utilizá-la. Há necessidade do envolvimento da cúpula organizacional, mas também dos gestores, para que sejam definidas e difundidas missão, visão, objetivos e estratégia, para todos os envolvidos e que se comprometam em montar um mapa estratégico específico para a realidade organizacional.

O uso de profissionais experientes e que conheçam bem a cultura organizacional, gestão estratégica e BSC é muito importante para o sucesso do processo, que pode ocorrer com o apoio de uma consultoria externa ou consultores internos devidamente qualificados, que pode representar o diferencial entre o sucesso e o fracasso da gestão estratégica.

O MPPE foi exitoso na escolha do formato definido para implantar o BSC no seu modelo de gestão estratégica, uma vez que a sequência de implantação seguida, adaptou-se à realidade da instituição. O projeto executivo previu dois eventos para públicos diferentes: palestra sobre gestão estratégica, com lideranças internas e externas; e um fórum de gestão estratégica, com diversos setores da sociedade, para formulação e aprimoramento do diagnóstico estratégico do MPPE, que optou por implantar o BSC paralelamente à definição de sua estratégia utilizada para o ciclo 2013-2016.

$\mathrm{Na}$ Gestão Estratégica 2013-2016, a metodologia BSC aplicada funcionou como ferramenta de gestão estratégia e como um sistema de avaliação de desempenho, estando alinhada com a classificação que Andrade \& Frazão (2011) estabeleceram para o BSC. Destaca-se, que este também é o entendimento da equipe de planejamento do MPPE, ao destacar nas entrevistas que, a metodologia BSC adotada pela organização, além de permitir avaliar desempenho, permite também, auxílio na tomada de decisão.

A execução da estratégia, por meio da realização de projetos estratégicos, foi considerada uma inovação pela gestão do MPPE. Dos seis projetos estratégicos realizados neste ciclo, a maioria atendeu aos objetivos, mesmo que para a equipe de planejamento da instituição, a utilização dos projetos estratégicos necessitará de adequações para o próximo ciclo, como, por exemplo, executar um projeto de cada vez e concluí-lo, antes de iniciar o próximo.

Outro aspecto identificado foi que o MPPE utilizou 72 (setenta e dois) indicadores para acompanhar o desempenho da instituição, considerado um número elevado, pois Kaplan $\&$ Norton $(1997,2010)$ indicam entre 20 e 25 para uso no BSC. A justificativa para o elevado número de indicadores está relacionada à natureza da organização, sua complexidade e atuação diversificada. No entanto, para o próximo ciclo estratégico, a equipe pretende reduzir esta quantidade, por reconhecer o excesso de indicadores utilizados.

A falta de apoio da alta administração das organizações tem sido destacada por diversos pesquisadores, como uma das principais razões para o insucesso na implantação do BSC. No MPPE, mesmo passando por 3 (três) Procuradores Gerais diferentes, durante o ciclo 2013-2016, a execução da estratégia não foi prejudicada. A assessoria de planejamento afirmou que os impactos causados pelas mudanças na direção da organização foram positivos. Para a equipe de planejamento, os projetos estratégicos, o painel de contribuição, os sistemas QlikView e Arquimedes tiveram grande parcela de contribuição para o avanço que a organização obteve no ciclo estratégico estudado. 
Portanto, o MPPE, pela primeira vez, teve um ciclo estratégico analisado desde a sua construção até a execução, com todas as etapas registradas e analisadas em sua essência e resultados alcançados. É uma iniciativa que já foi premiada nacionalmente, com o Prêmio Innovare, que tem como objetivo identificar, divulgar e difundir práticas que contribuam para o aprimoramento da Justiça no Brasil. Mesmo assim, deverá ser aprimorada para o novo ciclo de gestão estratégica organizacional, como evolução natural e estratégica de gestão.

Apesar do estudo ter se aprofundado na realidade analisada, as limitações em se tratar de um estudo de caso são reais e novas experiências podem ser desenvolvidas, com base na realidade identificada neste estudo. Pode-se até pensar em um estudo relativo à implantação da metodologia BSC, em outros Ministérios Públicos do Brasil, uma vez que, esta metodologia está sendo adotada em todo território brasileiro, por recomendação do Conselho Nacional do Ministério Público.

\section{REFERÊNCIAS}

Anagnostopoulos, K. P. \& Elmasides, G. (2010). Strategic Plan in a Greek Manufacturing Company: A Balanced Scorecard and Strategy Map Imlementation. International Journal of Business and Management, 5 (2), 12-25.

Andrade, I. R. S. \& Frazão, M. de F. A. (2011). Estratégia em ação: planejamento estratégico e Balanced Scorecard na OSID. Revista de Gestão, Finanças e Contabilidade, 1(1), 18 34.

Creswell, J.W. (2014). Investigação Qualitativa e Projeto de Pesquisa - Escolhendo Entre Cinco Abordagens - $3^{\mathrm{a}}$ Ed., 3 ed. Porto Alegre: Artmed.

Cunha, M. R. \& Kratz, L. (2016). Fatores críticos de sucesso no processo de implementação do Balanced Scorecard: um estudo de caso nas instituições federais de ensino superior. Revista de Ciências da Administração, 18(46), 96-108.

Dorweiler, V. \& Yakhou, M. (2005). Scorecard for academic administration performance on the campus. Managerial Auditing Journal, Bradford, 20(2), 138-144.

Felix, R., Felix, do P., \& Timóteo, R. (2011). Balanced Scorecard: adequação para a gestão estratégica nas organizações públicas. Revista do Serviço Público, 62(1), 51-74.

Fernandes, A. G., Furtado, R. P. M., \& Ferreira, P. A. (2016). Aplicação do Balanced Scorecard no auxílio à formulação do planejamento estratégico no setor público: o caso DAE/UFLA. E\&G Economia e Gestão. 16(42), 218-244.

Filgueiras, A. de A., Barros, Luana P. de S. \& Gomes, J. S. (2010). O processo de implantação do Balanced Scorecard em uma empresa estatal brasileira: o caso Petrobras. Revista de Gestão USP, São Paulo, 17(1), 45-57.

Galas, E. S. \& Forte, S. H. A. C. (2005). Fatores que interferem na implantação de um modelo de gestão estratégica baseado no Balanced Scorecard: estudo de caso em uma instituição pública. Revista de Administração Mackenzie, 6(2), 87-111.

Gallon, A.V., Nuintin, A. A., Curi, M. A. \& Nogueira, L. R. T. (2010). Implementação do Balanced Scorecard de acordo com os preceitos da gestão do conhecimento em uma organização que explora a atividade pecuária de gado bovino de corte em Minas Gerais. Revista Contemporânea de Contabilidade, UFSC, Florianópolis, 7(13), 63-88. 
Goldszmidt, R. G. B. (2003). Uma revisão de literatura dos fatores críticos para a implementação e uso do Balanced Scorecard. XXVII Encontro da ANPAD, Atibaia, SP, 20 a 24 de setembro, Brasil.

Gomes, R. C., Leal, A. C. S. \& Assis, V. A. (2013). Indicadores de desempenho para avaliar o desempenho de prefeituras municipais. TAC, Rio de Janeiro, 3(1), 1-15.

Kaplan, R.S., \& Norton, D. P. (1997). A estratégia em ação: Balanced Scorecard. Rio de Janeiro: Campus.

\section{. (2010). Conceptual foundations of the Balanced Scorecard. Boston, MA:} Harvard Business School.

Kartalis, N., Velentzas, J. \& Broni, G. (2013). Balanced Scorecard and performance measurement in a Greek Industry. Procedia Economics and Finance, 1(5), 413-422.

Litvac, B. T. C., Calhau, C. de F. S., Neto, O. L. S., \& Oliva, F. L. (2010). Avaliação do processo de implementação do Balanced Scorecard: um estudo de caso na indústria do cimento. Ver. Ciênc. Admin., 16(1), 33-53.

Martins, G. de A., \& Theóphilo, C. R. (2017). Investigação cientifica para ciências sociais aplicadas. $3^{\mathrm{a}}$. ed., São Paulo: Atlas.

Moreira, F. A. de L., Pessoa, M. N. M., Cabral, A. C. de A., Santos, S. M. dos, \& Roldan, V. P. S. (2012). Fatores críticos na implantação do Balanced Scorecard. Revista eletr. estrat., 5(3), 57-89.

Muller, J. R. (2001). Desenvolvimento do modelo de gestão aplicado à universidade, tendo por base o Balanced Scorecard. Dissertação de Mestrado. UFSC - Universidade Federal de Santa Catarina, Florianópolis - SC.

Muscat, A. R. N.; Biazzi, M. R. de; Miguel, P. A. C. (2007). Implementação da estratégia utilizando o BSC: um estudo de caso no setor público brasileiro. GEPROS - Gestão da produção, operações e sistemas, 2(3), 35-47.

Niven, P.R. (2003). Balanced Scorecard step-by-step for Government and Nonprofit Agencies. New Jersey: John Wiley \& Sons.

Northcott, D., \& Taulapapa, T. M. (2012). Using the Balanced Scorecard to manage performance in public sector organizations: issues and challenges. International Journal of Public Sector Management, 25(3), 166-191.

Odebrecht, G. V.; Giarola, P. G.; Silva, J. D.; Martins, C. \& Lima, C. R. M. (2013). Balanced Scorecard numa clínica de cirurgia plástica: uma proposta de ferramenta para gestão estratégica. Revista de Administração Hospitalar, 10(2), 13-29.

Osório, V. L. T. (2003). A utilização do Balanced Scorecard no aperfeiçoamento da administração pública gerencial: estudo de caso de uma autarquia municipal. Dissertação (Mestrado profissional em Gerência de Serviços) - Curso de Pós Graduação em Engenharia, Universidade Federal do Rio Grande do Sul, Porto Alegre, Brasil.

Peci, A., Pieranti, O. P., \& Rodrigues, S. (2008). Governança e New Public Management: convergências e contradições no contexto brasileiro. Revista $O \& S, 15(46), 39-55$.

Pernambuco. Ministério Público do Estado. (2014). Apresentação Monitoring the Strategy Map with QlikView, Assessoria de Planejamento e Estratégia Organizacional, Recife. 
. (2012). MPPE em FOCO, ano III, edição extra - Planejamento Estratégico,

Recife.

. (2014) Manual de gestão estratégica 2013/2016: Ministério Público de

Estado de Pernambuco; Recife: Procuradoria Geral de Justiça.

Pessoa, M. N. M. (2000). Gestão das universidades federais brasileiras - um modelo fundamentado no Balanced Scorecard. Tese (Doutorado em Engenharia de Produção) Programa de Pós-Graduação em Engenharia de Produção, Universidade Federal de Santa Catarina, Florianópolis, Brasil.

Rosa, M. M., Petri, S. M., Petri, L. R. F., \& Casagrande, M. D. H. (2014). O Balanced Scorecard como ferramenta de gestão estratégica e orçamentária da Fundação Cultural de Florianópolis. Revista Evidenciação Contábil \& Finanças, 2(1), 39-58.

Silva, P. D. A. da, Santos, O. M. dos \& Prochnik, V. (2008). Percepções quanto aos fatores críticos à implantação e uso do Balanced Scorecard: a experiência da Petrobras. In: Congresso Brasileiro de Contabilidade, Gramado, Brasil. Gramado: CFC 2008.

Souza, S. C. de \& Marinho, S. V. (2014). Planejamento estratégico baseado no balanced scorecard: um estudo de caso aplicado a uma pequena empresa de segurança. Gestão \& Planejamento, Salvador, 15(2), 213-237.

Schnorrenberger, D., Ambros, M. G., Gasparetto, V., \& Lunkes, R. J. (2015). Comparação entre métodos para avaliação de empresas. Revista de Gestão e Tecnologia, 5(1), 79-92.

Vargas, S. B. de, Diehl, C. A., Ayres, P. R. R.\& Monteiro, A. F. (2015). Indicadores nãofinanceiros de avaliação de desempenho: análise de conteúdo em relatórios de administração de empresas de telecomunicações. XV Congresso USP de Controladoria e Contabilidade, São Paulo, Brasil.

Vieira, J. G. S. (2010). Metodologia de pesquisa científica na prática. Curitiba: Editora Fael.

Wernke, R. \& Bornia, A.C. (2001). Balanced Scorecard: considerações e comentários. VIII Congresso Brasileiro de Custos, São Leopoldo/RS. São Leopoldo: Associação Brasileira de Custos, Brasil.

Wernke, R., Lunkes, R. J. \& Bornia, A. C. (2002). Balanced Scorecard: considerações acerca das dificuldades na implementação e das críticas ao modelo. IX Congresso Brasileiro de Custos, São Paulo/SP, Brasil. 